\title{
Quality of Life in Newly Diagnosed Moderate-to-Severe Ulcerative Colitis: Changes in the MOSAIK Cohort Over 1 Year
}

\author{
Ik Hyun Jo1, Kang-Moon Lee ${ }^{1}$, Dae Bum Kim¹, Ji Won Kim², Jun Lee³, Yoon Tae Jeen4, Tae-Oh Kim5, Joo Sung \\ $\mathrm{Kim}^{6}$, Jae Jun Park ${ }^{7}$, Sung Noh Hong ${ }^{8}$, Dong II Park ${ }^{9}$, Hyun-Soo Kim ${ }^{10}$, Yoo Jin Lee ${ }^{11}$, and Youngdoe Kim ${ }^{12}$ \\ ${ }^{1}$ Department of Internal Medicine, St. Vincent's Hospital, College of Medicine, The Catholic University of Korea, Suwon, ${ }^{2}$ Department \\ of Internal Medicine, SMG-SNU Boramae Medical Center, Seoul National University College of Medicine, Seoul, ${ }^{3}$ Department of \\ Internal Medicine, Chosun University Hospital, Gwangju, ${ }^{4}$ Department of Internal Medicine, Korea University Anam Hospital, Seoul, \\ ${ }^{5}$ Department of Internal Medicine, Inje University Haeundae Paik Hospital, Busan, ${ }^{6}$ Department of Internal Medicine and Liver \\ Research Institute, Seoul National University College of Medicine, ${ }^{7}$ Department of Internal Medicine, Severance Hospital, Yonsei \\ University College of Medicine, ${ }^{8}$ Department of Internal Medicine, Samsung Medical Center, ${ }^{9}$ Department of Internal Medicine, \\ Kangbuk Samsung Hospital, Seoul, ${ }^{10}$ Department of Internal Medicine, Wonju Severance Christian Hospital, Wonju, ${ }^{11}$ Department of \\ Internal Medicine, Keimyung University Dongsan Hospital, Daegu, and ${ }^{12}$ Clinical Research Science Team, Medical Affairs, Janssen \\ Korea Ltd., Seoul, Korea
}

See editorial on page 317.

\section{Article Info}

Received March 30, 2021

Revised May 10, 2021

Accepted June 14, 2021

Published online August 11, 2021

\section{Corresponding Author}

Kang-Moon Lee

ORCID https://orcid.org/0000-0003-2850-4553

E-mail drmaloman@catholic.ac.kr
Background/Aims: Improving quality of life has been gaining importance in ulcerative colitis (UC) management. The aim of this study was to investigate changes in health-related quality of life (HRQL) and related factors in patients with moderate-to-severe UC.

Methods: A multicenter, hospital-based, prospective study was performed using a Moderateto-Severe Ulcerative Colitis Cohort in Korea (the MOSAIK). Changes in HRQL, evaluated using the 12-Item Short Form Health Survey (SF-12) and Inflammatory Bowel Disease Questionnaire (IBDQ), were analyzed at the time of diagnosis and 1 year later.

Results: In a sample of 276 patients, the mean age was 38.4 years, and the majority of patients were male (59.8\%). HRQL tended to increase in both the IBDQ and SF-12 1 year after diagnosis. A higher partial Mayo score was significantly related to poorer HRQL on the IBDQ and SF-12 in a linear mixed model $(p<0.01)$. Inflammatory markers such as $C$-reactive protein $(C R P)$ or erythrocyte sedimentation rate also showed a negative correlation on HRQL $(p<0.05)$. Patients whose IBDQ score improved by 16 or more $(71.2 \%)$ in 1 year were younger, tended to be nonsmokers, and had a lower partial Mayo score and CRP than those whose IBDQ score did not. There was no significant association between $\mathrm{HRQL}$ and disease extent, treatments at diagnosis, or the highest treatment step during the 1-year period.

Conclusions: Optimally controlled disease status improves HRQL in patients with moderate-tosevere UC. The partial Mayo score and inflammatory markers may be potential indicators reflecting the influence of UC on patient's daily lives. (Gut Liver 2022;16:384-395)

Key Words: Colitis, ulcerative; Quality of life; Treatment outcome; Cohort studies

\section{INTRODUCTION}

Ulcerative colitis (UC) is a chronic disease that involves repeated exacerbation and remission while showing nonspecific inflammation of the colonic mucosa. ${ }^{1}$ Patients may experience difficulties in daily life due to frequent symptoms such as diarrhea, bloody stool, stool urgency, and abdominal pain. They can suffer from various adverse events as a result of treatments, ranging from mild to lifethreatening. ${ }^{2-4}$ Since the disease course requires patients to receive constant treatment, it can interfere with their daily performance and ability to work. ${ }^{5,6}$ Causing not only physical and social problems, UC can be also harmful to mental health. Repeated cycles of remission and exacerbation cause patients to fear recurrence, resulting in anxiety, depression, and even social isolation. ${ }^{7,8}$ In chronic diseases 
such as UC, all these physical, psychological, and social dysfunctions eventually impact a patient's quality of life. The concept of quality of life has been gaining importance not only in terms of compliance, but also as a crucial goal of treatment. ${ }^{9,10}$ Considering that UC is a difficult disease to cure, the goal of treatment for UC should be to improve the quality of life of the patient, and not just provide symptom relief. ${ }^{11}$ Since adherence to treatment for chronic diseases is affected by the quality of life, ${ }^{12}$ quality of life should be emphasized during the treatment process.

Moderate-to-severe UC patients with severe endoscopic mucosal findings at the time of diagnosis are expected to have a worse prognosis, such as a more aggressive progression, poorer response to medical therapy, and greater need for surgery than those who are not. ${ }^{13}$ Accordingly, a high standard of treatment and monitoring is required in managing moderate-to-severe UC patients. A patient's experience of rapid improvement in symptoms and subsequent improvement in quality of life in the initial phase of treatment may have a positive effect on adherence to treatment, and furthermore, the future treatment prognosis. Therefore, it is worthwhile to pay attention to the early pattern of quality of life and its changes in moderate-to-severe UC patients. This study aimed to investigate changes in healthrelated quality of life (HRQL) of patients with moderateto-severe UC, based on 1-year follow-up cohort data. We also tried to identify any related factors that may contribute to HRQL improvements in the early period after diagnosis.

\section{MATERIALS AND METHODS}

\section{The MOSAIK cohort}

The MOSAIK cohort is a nationwide, multicenter, prospective, observational cohort of moderate-to-severe UC that includes participants from 30 teaching hospitals in Korea. Patients who met the following criteria were enrolled in the cohort: (1) age 7 or older; (2) newly diagnosed with moderate-to-severe UC; (3) diagnosed at a tertiary referral hospital within 4 weeks from the visit or diagnosed at a primary or secondary referral hospital with subsequent confirmation by a tertiary center within 8 weeks; and (4) informed consent. Those who had participated in other interventional clinical trials with biologics, corticosteroids, or other medications for UC, had a history of colectomy, or no definite diagnosis of UC were excluded. ${ }^{8,14}$

Patients were diagnosed with UC when met at least three of the following four criteria: (1) symptoms such as abdominal pain, diarrhea, hematochezia, or pus in stools; (2) endoscopic findings of continuous mucosal inflammation, which involve rectum and may include part or all of the colon; (3) microscopic findings consistent with UC; and (4) the exclusion of Crohn's disease and indeterminate colitis. ${ }^{8}$ The severity of UC was classified according to the Mayo scale. A total Mayo score of 6 to 10 was classified as moderate, and a score of 11 or higher was classified as severe UC. ${ }^{15}$

This study complies with the Declaration of Helsinki, and written consent was obtained from each enrolled patient. The study was registered at www.clinicaltrials. gov (ClinicalTrials.gov identifier: NCT02229344) and was approved by the institutional review boards of every participating medical institution (approval number: VC13ESME0219).

\section{Evaluation of $H R Q L$}

HRQL at the time of diagnosis and 1 year after diagnosis was investigated using two questionnaires: the Inflammatory Bowel Disease Questionnaire (IBDQ) and the 12-Item Short Form Health Survey (SF-12). IBDQ is a tool to measure disease-specific HRQL for patients with inflammatory bowel disease. It consists of 32 items in four dimensions, and the total score ranges from 32 to 224 . A higher IBDQ score indicates better HRQL. We used a verified Korean version of the IBDQ, which has been used in several previous studies. ${ }^{16}$ Since Gregor et al. had claimed an increase of IBDQ score by 16 or higher as a criterion for meaningful HRQL improvement, this criterion has been used by other researchers. ${ }^{17-19}$ We investigated whether the IBDQ score of the total cohort rose by 16 or more after 1 year from diagnosis.

The SF-12 is a well-known generic HRQL measuring tool that is simplified by selecting only 12 items from the SF-36. Eight health concepts can be scored according to the results of the self-reported questionnaire. By calculating eight scores with a certain method, a total score between 0 and 100 is obtained, where a higher score indicates a better quality of life. ${ }^{20,21}$ In addition, eight individual scores were divided into two groups and used to measure physical component summary (PCS) and mental component summary (MCS). ${ }^{22}$

\section{Clinical variables of the subjects}

The MOSAIK cohort collects standardized clinical data through periodic follow-up visits for 5 years from baseline at the time of diagnosis of participating patients. During the follow-up period, self-reported questionnaires, regular colonoscopies, and blood tests were conducted according to the study plan. HRQL results and demographic information were investigated. The patient's health burden due to comorbidity was calculated using the Charlson comorbidity index. ${ }^{23}$ Other UC-related factors were also investigated: the extent of the disease, disease activity, relevant 
laboratory findings, and the details of the treatment process. Because a patient's treatment may fluctuate throughout the 1-year period, we analyzed UC treatments from only two perspectives: the highest treatment step during the 1-year period and the UC treatment at each visit (baseline and 1 year after diagnosis). The highest treatment step was defined as the higher treatment level from four therapeutic potency levels: 5 -aminosalicylic acid, corticosteroid, immune modulators, and biologics. ${ }^{24}$ The UC treatment at each visit was defined as the type of the highest treatment step during the 12 weeks before visit.

\section{Statistical methods}

Linear mixed effects regression models, which have been recommended for analyzing longitudinal outcomes, were used to assess the effects of each demographic and clinical characteristic on the outcomes (IBDQ, SF-12

\section{Enrolled patients}

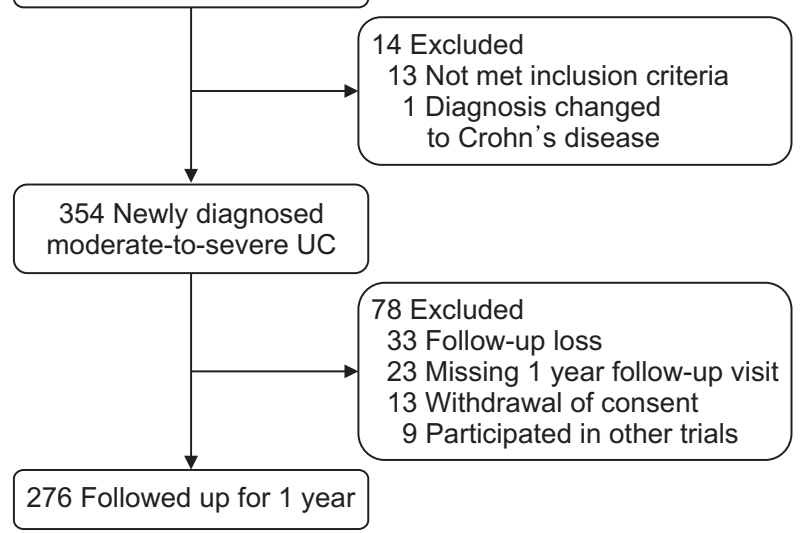

Fig. 1. Flowchart for the composition of the study population. UC, ulcerative colitis.

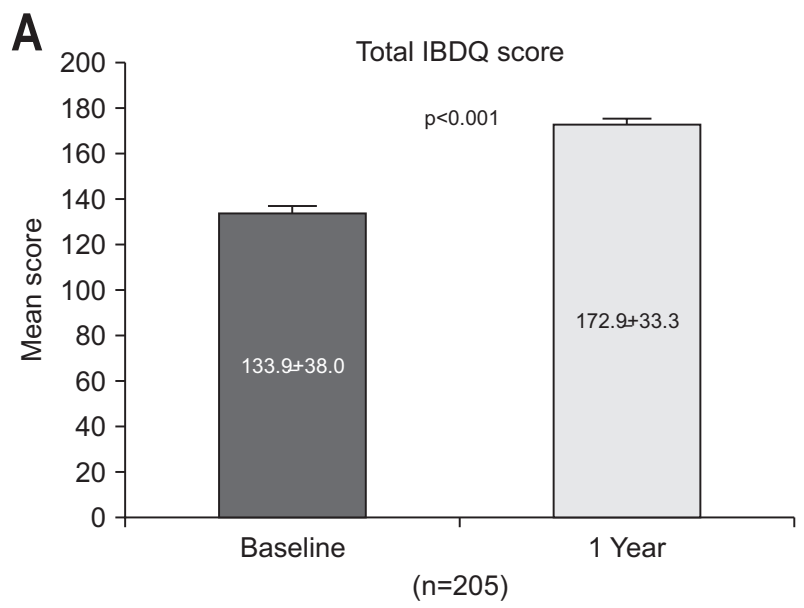

[MCS/PCS] scores) over time (baseline and 1 year). For all mixed effects models, the confounding covariates of age, sex, time, and partial Mayo score at each visit were included as fixed effects. For each independent variable, demographic or clinical characteristics, individual models using fixed effects and each independent variable were fitted, and independent variables that had an overall p-value $\geq 0.1$ from the individual model were not included in the final model to ensure a parsimonious model. For considering multicollinearity of laboratory independent variables in the final model, representative variables were selected by correlation coefficients between variables and used for the final model.

p-values less than 0.05 were considered statistically significant. All analyses were performed using the statistical software package SAS 9.4 (Statistical Analysis System, SASInstitute, Cary, NC, USA).

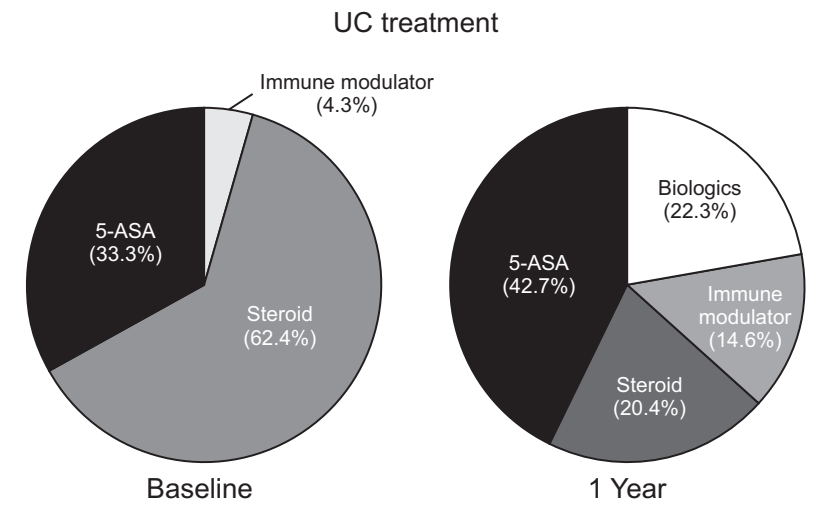

Fig. 2. Proportion of the highest treatment step at each time point lbaseline and 1 year after diagnosis).

UC, ulcerative colitis; 5-ASA, 5-aminosalicylic acid.

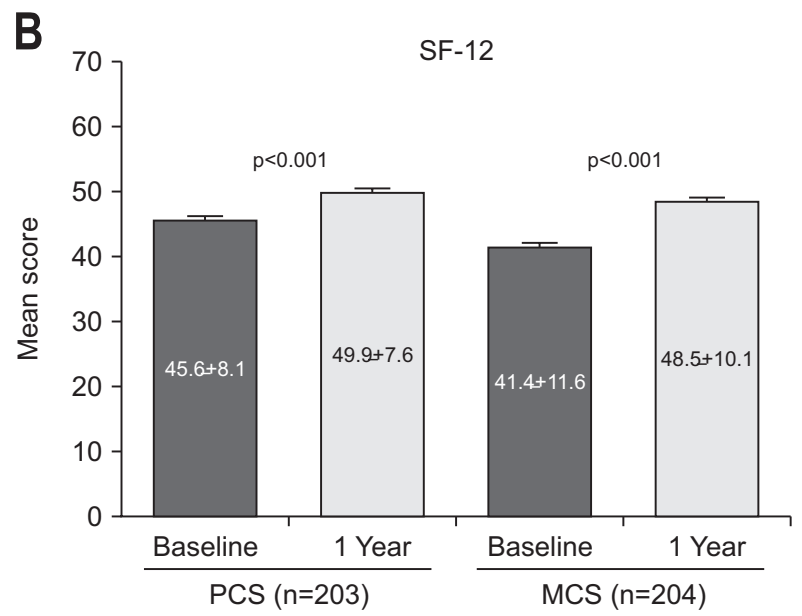

Fig. 3. Differences in health-related quality of life indices between baseline and after 1 year. (A) Inflammatory Bowel Disease Questionnaire (IBDQ) score and (B) 12-Item Short Form Health Survey (SF-12). Data are presented as the mean $\pm S D$. PCS, physical component summary; MCS, mental component summary. 


\section{RESULTS}

\section{Characteristics of the study cohort}

A total of 368 patients were enrolled in the cohort, and 354 patients were finally diagnosed with moderateto-severe UC. Of these, 78 patients were excluded, and a total of 276 patients were analyzed (Fig. 1). The mean age of the patients was 38.4 years, and there were more male patients than female patients. The majority of the patients had left-sided or extensive colitis (E2 and E3), and about a third had endoscopically severe disease. Within 12 weeks before the time of cohort enrollment, steroids were most

Table 1. Demographic and Clinical Characteristics of the Study Population at Baseline and the 1-Year Follow-up

\begin{tabular}{|c|c|c|c|}
\hline Category (n=276) & No. of baseline/No. of $1 \mathrm{yr}$ & Baseline & $1 \mathrm{yr}$ \\
\hline Age, yr & 276 & $38.4 \pm 15.6$ & \\
\hline Sex & 276 & & \\
\hline Male & & 165 (59.8) & \\
\hline Female & & $111(40.2)$ & \\
\hline $\mathrm{BMI}, \mathrm{kg} / \mathrm{m}^{2}$ & $266 / 209$ & $22.3 \pm 3.1$ & $23.1 \pm 3.2$ \\
\hline Drinking history & $269 / 201$ & & \\
\hline Never & & $73(27.1)$ & $52(25.9)$ \\
\hline Current & & $114(42.4)$ & 81 (40.3) \\
\hline Former & & 82 (30.5) & 68 (33.8) \\
\hline Smoking history & $267 / 200$ & & \\
\hline Never & & 144 (53.9) & $110(55.0)$ \\
\hline Current & & $24(9.0)$ & 19 (9.5) \\
\hline Former & & 99 (37.1) & 71 (35.5) \\
\hline $\mathrm{CCl}$ score & $260 / 253$ & $0.15 \pm 0.60$ & $0.07 \pm 0.31$ \\
\hline Disease extent & $270 / 105$ & & \\
\hline E1 (proctitis) & & 29 (10.7) & $13(12.4)$ \\
\hline E2 (left-sided colitis) & & $125(46.3)$ & 44 (41.9) \\
\hline E3 (extensive colitis) & & $116(43.0)$ & $48(45.7)$ \\
\hline Partial Mayo score & $276 / 276$ & $5.8 \pm 1.4$ & $1.6 \pm 1.9$ \\
\hline Endoscopy & $276 / 86$ & & \\
\hline Normal & & 0 & $20(23.3)$ \\
\hline Mild disease & & 0 & 30 (34.9) \\
\hline Moderate disease & & 195 (70.7) & $27(31.4)$ \\
\hline Severe disease & & 81 (29.3) & 9 (10.5) \\
\hline \multicolumn{4}{|l|}{ Laboratory test } \\
\hline $\mathrm{ESR}, \mathrm{mm} / \mathrm{hr}$ & $237 / 168$ & $20.8 \pm 18.2$ & $15.4 \pm 17.1$ \\
\hline $\mathrm{CRP}, \mathrm{mg} / \mathrm{dL}$ & $247 / 195$ & $2.8 \pm 7.9$ & $1.0 \pm 3.6$ \\
\hline WBC, $10^{3} / \mu \mathrm{L}$ & $268 / 200$ & $12.2 \pm 60.1$ & $6.7 \pm 2.2$ \\
\hline Hemoglobin, g/dL & $269 / 200$ & $12.7 \pm 2.1$ & $14.2 \pm 7.0$ \\
\hline Albumin, g/dL & $238 / 148$ & $4.0 \pm 0.6$ & $4.3 \pm 0.4$ \\
\hline UC treatment* & $93 / 103$ & & \\
\hline 5-ASA & & 31 (33.3) & $44(42.7)$ \\
\hline Steroid & & $58(62.4)$ & $21(20.4)$ \\
\hline Immune modulator & & $4(4.3)$ & $15(14.6)$ \\
\hline Biologics & & 0 & 23 (22.3) \\
\hline Highest treatment during the $1 \mathrm{yr}$ & 276 & & \\
\hline 5-ASA & & & $71(25.7)$ \\
\hline Steroid & & & 87 (31.5) \\
\hline Immune modulator & & & 66 (23.9) \\
\hline Biologics & & & 52 (18.8) \\
\hline Total IBDQ score & $274 / 207$ & $136.7 \pm 38.2$ & $173.2 \pm 33.3$ \\
\hline Change of IBDQ score $\geq 16$ from baseline & 205 & & 146 (71.2) \\
\hline \multicolumn{4}{|l|}{ SF-12 } \\
\hline MCS & $274 / 206$ & $41.9 \pm 11.5$ & $48.5 \pm 10.1$ \\
\hline PCS & $273 / 206$ & $45.9 \pm 8.3$ & $49.9 \pm 7.6$ \\
\hline
\end{tabular}

Data are presented as the mean \pm SD or number $(\%)$.

$\mathrm{BMI}$, body mass index; CCI, Charlson comorbidity index; ESR, erythrocyte sedimentation rate; CRP, C-reactive protein; WBC, white blood cell; UC, ulcerative colitis; 5-ASA, 5-aminosalicylic acid; IBDQ, Inflammatory Bowel Disease Questionnaire; SF-12, 12-Item Short Form Health Survey; MCS, mental component summary; PCS, physical component summary.

*Highest UC treatment during 12 weeks before each visit (baseline or 1 year). 
frequently used as the highest treatment step, and no biologics were used. One year after the baseline, the highest treatment step mainly consisted of 5-aminosalicylic acid or biologics (Fig. 2). The partial Mayo score decreased dramatically at 1 year from baseline, and alleviation of inflammatory markers was confirmed. Both the IBDQ and SF- 12 scores were also increased, indicating an improvement in HRQL through 1 year of treatment after enrollment (Fig. 3). However, the mean final SF-12 score did not exceed 50, and the score increase was less than that of the IBDQ. Details of the study cohort characteristics are listed in Table 1.

\section{Clinical factors influencing changes of $H R Q L$}

During the year of follow-up, women tended to have lower IBDQ scores than men $(\mathrm{p}=0.059)$. A higher partial Mayo score was significantly related to lower HRQL. In the individual model, right colon involvement, a history of alcohol consumption, and increased inflammatory markers (white blood cell count, erythrocyte sedimentation rate
[ESR], and C-reactive protein [CRP]) were all associated with a deterioration of HRQL, whereas hemoglobin and albumin levels were positively associated with HRQL. In the final model, higher partial Mayo scores, history of alcohol consumption, and higher CRP were significantly correlated with low IBDQ scores (Table 2).

The SF-12 score was negatively associated with the partial Mayo score in both MCS and PCS. In MCS, the partial Mayo score and ESR showed a negative correlation in the final model (Table 3), whereas the partial Mayo score, patient age, alcohol consumption history, serum CRP, and white blood cell count had negative effects on the PCS. High serum hemoglobin level showed a positive correlation with the PCS (Table 4).

\section{Differences between patient groups according to IBDQ improvement}

The IBDQ scores at the time of diagnosis and 1 year after diagnosis were confirmed in 205 subjects. In 146

Table 2. Results from Linear Mixed Effects Models for IBDQ

\begin{tabular}{|c|c|c|c|c|c|c|c|}
\hline \multirow{2}{*}{ Variable } & \multirow{2}{*}{ Reference } & \multicolumn{3}{|c|}{ IBDQ individual model } & \multicolumn{3}{|c|}{ IBDQ final model } \\
\hline & & Beta & SE & $p$-value & Beta & SE & p-value \\
\hline Age & & - & - & - & 0.02 & 0.135 & 0.908 \\
\hline Sex (female) & Male & - & - & - & -8.95 & 4.711 & 0.059 \\
\hline Partial Mayo score & & - & - & - & -6.99 & 1.222 & $<0.001$ \\
\hline Visit & & - & - & - & 1.12 & 1.594 & 0.485 \\
\hline Disease extent (E2, left-sided colitis) & E1 & -1.65 & 6.398 & 0.797 & 1.53 & 7.015 & 0.828 \\
\hline Disease extent (E3, extensive colitis) & & -12.85 & 6.597 & 0.052 & -5.87 & 7.217 & 0.417 \\
\hline Alcohol (current) & Never & -9.47 & 4.381 & 0.031 & -12.09 & 5.408 & 0.026 \\
\hline Alcohol (former) & & -12.35 & 4.490 & 0.006 & -11.89 & 5.711 & 0.038 \\
\hline Laboratory test (ESR) & - & -0.25 & 0.101 & 0.012 & - & - & - \\
\hline Laboratory test (CRP) & - & -0.66 & 0.245 & 0.008 & -0.61 & 0.269 & 0.023 \\
\hline Laboratory test (WBC) & - & -0.06 & 0.032 & 0.054 & -0.06 & 0.034 & 0.061 \\
\hline Laboratory test (hemoglobin) & - & 0.57 & 0.306 & 0.062 & 1.40 & 1.113 & 0.210 \\
\hline Laboratory test (albumin) & - & 10.48 & 3.219 & 0.001 & - & - & - \\
\hline
\end{tabular}

IBDQ, Inflammatory Bowel Disease Questionnaire; SE, standard error; ESR, erythrocyte sedimentation rate; CRP, C-reactive protein; WBC, white blood cell.

Table 3. Results from Linear Mixed Effects Models for SF-12 (MCS)

\begin{tabular}{|c|c|c|c|c|c|c|c|}
\hline \multirow{2}{*}{ Variable } & \multirow{2}{*}{ Reference } & \multicolumn{3}{|c|}{ MCS individual model } & \multicolumn{3}{|c|}{ MCS final model } \\
\hline & & Beta & SE & p-value & Beta & SE & $\mathrm{p}$-value \\
\hline Age & & - & - & - & 0.05 & 0.043 & 0.223 \\
\hline Sex (female) & Male & - & - & - & -2.76 & 1.385 & 0.047 \\
\hline Partial Mayo score & & - & - & - & -1.41 & 0.395 & $<0.001$ \\
\hline Visit & & - & - & - & 0.06 & 0.530 & 0.906 \\
\hline Disease extent (E2, left-sided colitis) & E1 & -1.93 & 2.000 & 0.335 & -1.41 & 2.251 & 0.533 \\
\hline Disease extent (E3, extensive colitis) & & -4.34 & 2.052 & 0.035 & -3.43 & 2.296 & 0.137 \\
\hline Laboratory test (ESR) & - & -0.06 & 0.032 & 0.045 & -0.08 & 0.038 & 0.028 \\
\hline Laboratory test (hemoglobin) & - & 0.17 & 0.099 & 0.094 & - & - & - \\
\hline Laboratory test (albumin) & - & 1.99 & 1.047 & 0.059 & - & - & - \\
\hline
\end{tabular}

SF-12, 12-Item Short Form Health Survey; MCS, mental component summary; SE, standard error; ESR, erythrocyte sedimentation rate. 
Table 4. Results from Linear Mixed Effects Models for SF-12 (PCS)

\begin{tabular}{|c|c|c|c|c|c|c|c|}
\hline \multirow{2}{*}{ Variable } & \multirow{2}{*}{ Reference } & \multicolumn{3}{|c|}{ PCS individual model } & \multicolumn{3}{|c|}{ PCS final model } \\
\hline & & Beta & SE & $\mathrm{p}$-value & Beta & SE & $\mathrm{p}$-value \\
\hline Age & & - & - & - & -0.08 & 0.031 & 0.009 \\
\hline Sex (female) & Male & - & - & - & -0.04 & 1.061 & 0.973 \\
\hline Partial Mayo score & & - & - & - & -0.72 & 0.264 & 0.007 \\
\hline Visit & & - & - & - & 0.02 & 0.337 & 0.954 \\
\hline Alcohol (current) & Never & 0.81 & 1.031 & 0.432 & -0.80 & 1.215 & 0.513 \\
\hline Alcohol (former) & & -1.62 & 1.053 & 0.125 & -3.03 & 1.273 & 0.018 \\
\hline Disease extent (E2, left-sided colitis) & E1 & 0.63 & 1.461 & 0.668 & 1.69 & 1.557 & 0.279 \\
\hline Disease extent (E3, extensive colitis) & & -2.61 & 1.512 & 0.085 & -0.71 & 1.608 & 0.657 \\
\hline Laboratory test (ESR) & - & -0.09 & 0.023 & $<0.001$ & - & - & - \\
\hline Laboratory test (CRP) & - & -0.18 & 0.057 & 0.001 & -0.14 & 0.059 & 0.023 \\
\hline Laboratory test (WBC) & - & -0.02 & 0.007 & 0.028 & -0.02 & 0.007 & 0.027 \\
\hline Laboratory test (hemoglobin) & - & 0.13 & 0.071 & 0.081 & 0.63 & 0.245 & 0.011 \\
\hline Laboratory test (albumin) & - & 2.98 & 0.741 & $<0.001$ & - & - & - \\
\hline
\end{tabular}

SF-12, 12-Item Short Form Health Survey; PCS, physical component summary; SE, standard error; ESR, erythrocyte sedimentation rate; CRP, Creactive protein; $\mathrm{WBC}$, white blood cell.

patients (71.2\%), the IBDQ score rose above 16 and this group tended to be younger than the group that did not. The proportion of those with a smoking history was also smaller in the former group, and the baseline partial Mayo score was significantly lower. CRP and serum albumin differed according to the degree of change in the IBDQ score: CRP was lower and serum albumin was higher in the group with an IBDQ score change of 16 or more. As for SF-12, there was no difference in MCS and PCS scores between two groups (Table 5).

We performed a subgroup analysis according to baseline disease extent and severity. When the disease extent was divided into two groups (E1+E2 vs E3), results were similar to those of total study population. In the subgroup analysis according to baseline severity, the severe group showed a significant difference in the highest treatment step during the 1-year follow-up. Notably, the proportion of biologics use was higher in the group whose IBDQ score did not improve by 16 (41.7\% vs $13.2 \%)$. However, for the highest UC treatment within 12 weeks from 1 year after diagnosis, biologics were used more often in the better IBDQ improvement group (26.1\% vs $12.5 \%$ ), but the difference was not statistically significant (Table 6).

\section{DISCUSSION}

The treatment response and subjective experience of a patient in the early phase of disease can affect adherence to long-term treatment, especially in chronic diseases such as UC. ${ }^{25}$ Therefore, we focused on HRQL in the early stages of UC. In this study, we confirmed that improvement of HRQL can be achieved through consistent treatment for 1 year, even in patients with moderate-to-severe UC. A large-scale, population-based study conducted in Europe also reported increasing HRQL for 1 year, similar to our result. ${ }^{26}$

Many studies have indicated that patients with IBD suffer a decline in quality of life compared to the non-patient population. ${ }^{27-29}$ However, it is suggested in several studies that the quality of life of patients is not always inferior to the general population. ${ }^{30,31}$ These different outcomes might arise in the study design: the quality of life was not usually different from the non-patient population in studies with long-term follow-up for more than several years. The proportion of patients whose disease is well controlled and in remission may have increased over a long time from the first diagnosis, and this may be reflected in the average quality of life for IBD patients. Possible evidence for this point was also found in our results. Even after a short follow-up period of 1 year, both disease-specific HRQL and generic HRQL of patients improved in our data. However, SF-12 did not reach 50 points after 1 year in MCS and PCS, and the increase was insignificant compared to IBDQ. This means that it may take a certain amount of time to achieve significant improvement in generic HRQL. Overall, preceding studies and our results suggest that, while patients with moderate-to-severe UC may face exacerbations in the disease course, their HRQL can be improved up to similar level of the non-patient population with appropriate treatment.

Interestingly, our study showed that the percentage of biologics among the highest treatment step received in 1 year was higher in the severe UC group who did not have any significant improvement (16 or over) of IBDQ for 1 year. A similar trend was also observed in a large-scale European study: poorer perception of HRQL was confirmed in UC patients who had been administered biologics 
Table 5. Clinical Characteristics of the Study Population at the 1-Year Follow-up According to the IBDQ Response

\begin{tabular}{|c|c|c|c|c|}
\hline Variable & $n^{\prime}$ & $\begin{array}{c}\text { Change IBDQ score } \\
\text { from baseline }<16(n=59)\end{array}$ & $\begin{array}{c}\text { Change IBDQ score } \\
\text { from baseline } \geq 16(n=146)\end{array}$ & p-value \\
\hline Age, yr & $59 / 146$ & $42.9 \pm 16.2$ & $37.9 \pm 15.1$ & 0.037 \\
\hline Sex (female) & $59 / 146$ & $21(35.6)$ & 67 (45.9) & 0.178 \\
\hline $\mathrm{BMI}, \mathrm{kg} / \mathrm{m}^{2}$ & $47 / 119$ & $23.1 \pm 3.2$ & $23.1 \pm 3.3$ & 0.956 \\
\hline Drinking history & $58 / 141$ & & & 0.495 \\
\hline Never & & $14(24.1)$ & $38(27.0)$ & \\
\hline Current & & $27(46.6)$ & $53(37.6)$ & \\
\hline Former & & $17(29.3)$ & 50 (35.5) & \\
\hline Smoking history & $58 / 140$ & & & 0.017 \\
\hline Never & & $26(44.8)$ & 83 (59.3) & \\
\hline Current & & 3 (5.2) & $16(11.4)$ & \\
\hline Former & & 29 (50.0) & 41 (29.3) & \\
\hline $\mathrm{CCl}$ score & $57 / 136$ & $0.11 \pm 0.41$ & $0.07 \pm 0.31$ & 0.749 \\
\hline Disease extent & $32 / 55$ & & & 0.344 \\
\hline E1 (proctitis) & & $6(18.8)$ & $5(9.1)$ & \\
\hline E2 (left-sided colitis) & & $13(40.6)$ & $21(38.2)$ & \\
\hline E3 (extensive colitis) & & $13(40.6)$ & 29 (52.7) & \\
\hline Partial Mayo score & $59 / 146$ & $2.4 \pm 2.2$ & $1.5 \pm 1.8$ & 0.005 \\
\hline Endoscopy & $26 / 47$ & & & 0.143 \\
\hline Normal & & $3(11.5)$ & 14 (29.8) & \\
\hline Mild disease & & 8 (30.8) & 18 (38.3) & \\
\hline Moderate disease & & 12 (46.2) & $11(23.4)$ & \\
\hline Severe disease & & $3(11.5)$ & 4 [8.5] & \\
\hline \multicolumn{5}{|l|}{ Laboratory test } \\
\hline ESR, $\mathrm{mm} / \mathrm{hr}$ & $30 / 103$ & $20.7 \pm 19.5$ & $14.0 \pm 15.6$ & 0.069 \\
\hline $\mathrm{CRP}, \mathrm{mg} / \mathrm{dL}$ & $36 / 116$ & $2.1 \pm 6.2$ & $0.7 \pm 2.7$ & 0.002 \\
\hline WBC, $10^{3} / \mu \mathrm{L}$ & $38 / 117$ & $7.1 \pm 2.6$ & $6.6 \pm 2.2$ & 0.368 \\
\hline Hemoglobin, g/dL & $38 / 117$ & $13.5 \pm 1.6$ & $14.5 \pm 9.1$ & 0.646 \\
\hline Albumin, g/dL & $30 / 83$ & $4.1 \pm 0.5$ & $4.4 \pm 0.3$ & 0.004 \\
\hline UC treatment* & $26 / 53$ & & & 0.560 \\
\hline 5-ASA & & $12(46.2)$ & 18 (34.0) & \\
\hline Steroid & & $7(26.9)$ & $12(22.6)$ & \\
\hline Immune modulator & & $3(11.5)$ & $10(18.9)$ & \\
\hline Biologics & & $4(15.4)$ & $13(24.5)$ & \\
\hline Highest treatment during the $1 \mathrm{yr}$ & $59 / 146$ & & & 0.105 \\
\hline 5-ASA & & $14(23.7)$ & $30(20.5)$ & \\
\hline Steroid & & $13(22.0)$ & 50 (34.2) & \\
\hline Immune modulator & & $15(25.4)$ & $43(29.5)$ & \\
\hline Biologics & & $17(28.8)$ & 23 (15.8) & \\
\hline Total IBDQ score & $59 / 146$ & $156.4 \pm 41.1$ & $179.6 \pm 27.0$ & $<0.001$ \\
\hline \multicolumn{5}{|l|}{ SF-12 } \\
\hline MCS & $58 / 146$ & $46.9 \pm 10.3$ & $49.1 \pm 10.0$ & 0.151 \\
\hline PCS & $58 / 146$ & $49.1 \pm 8.4$ & $50.2 \pm 7.3$ & 0.605 \\
\hline
\end{tabular}

Data are presented as the mean \pm SD or number $(\%) . n$ : number of subjects in the analysis set; $n^{\prime}:$ number of available subjects per variable; percentages were based on $\mathrm{n}^{\prime}$.

IBDQ, Inflammatory Bowel Disease Questionnaire; BMI, body mass index; CCl, Charlson comorbidity index; ESR, erythrocyte sedimentation rate; CRP, C-reactive protein; WBC, white blood cell; UC, ulcerative colitis; 5-ASA, 5-aminosalicylic acid; SF-12, 12-Item Short Form Health Survey; MCS, mental component summary; PCS, physical component summary.

*Highest UC treatment during 12 weeks before each visit (baseline or 1 year).

within the first 1 year of the disease. Thus, it may appear as if the use of biologics is associated with poorer HRQL. However, we think that the reason for these results is that the HRQL was poorer in those who had a severe clinical course requiring biologics despite the relatively early stage of the disease. As evidence of our assumption, our data showed that the proportion of patients using biologics was higher in the group with an IBDQ score of 16 or more for the aspect of the highest ongoing treatment step at the point of 1 year. This result suggests that biologics may have a favorable action on HRQL in patients with early moderate-to-severe UC. In fact, in several studies currently 


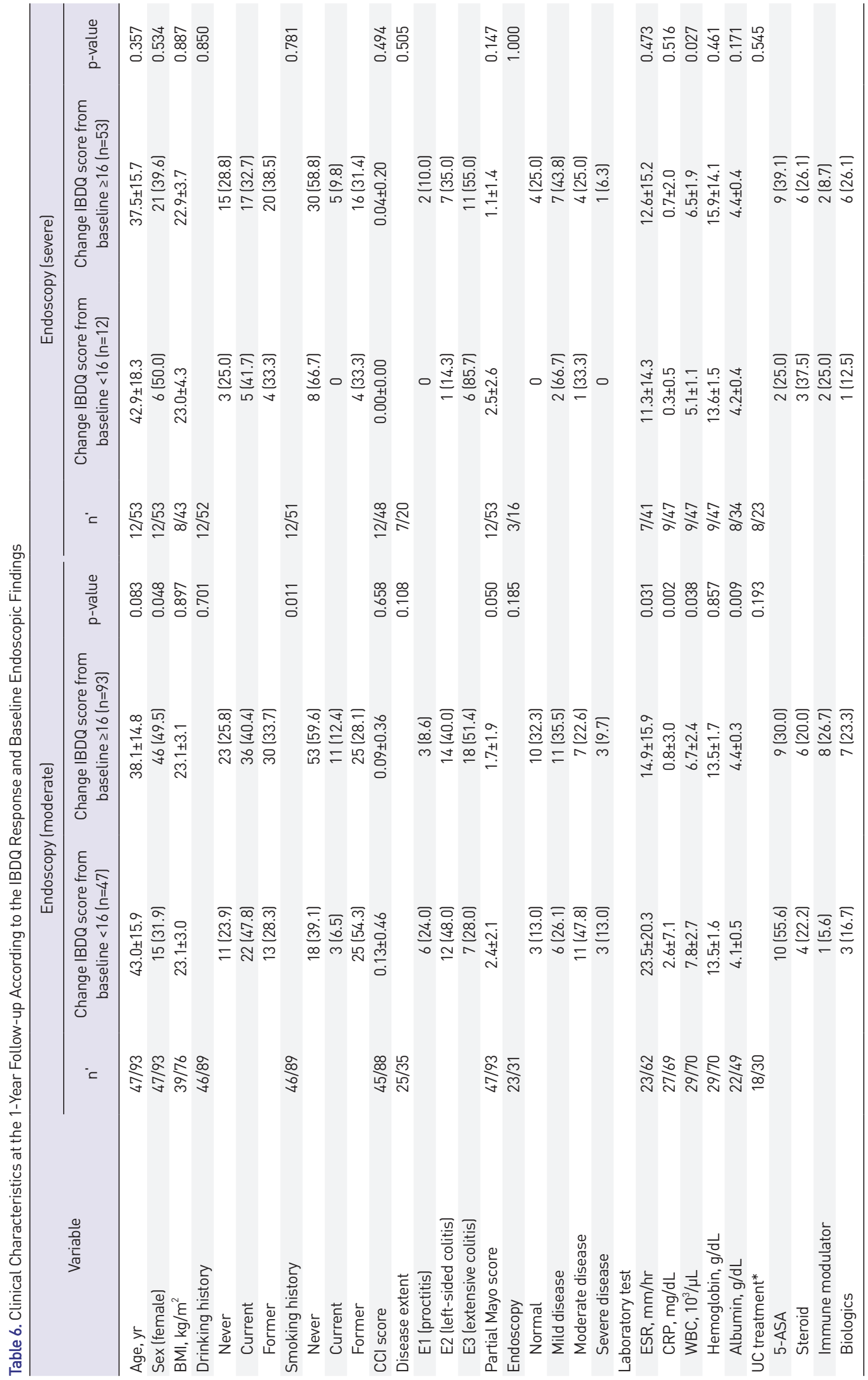




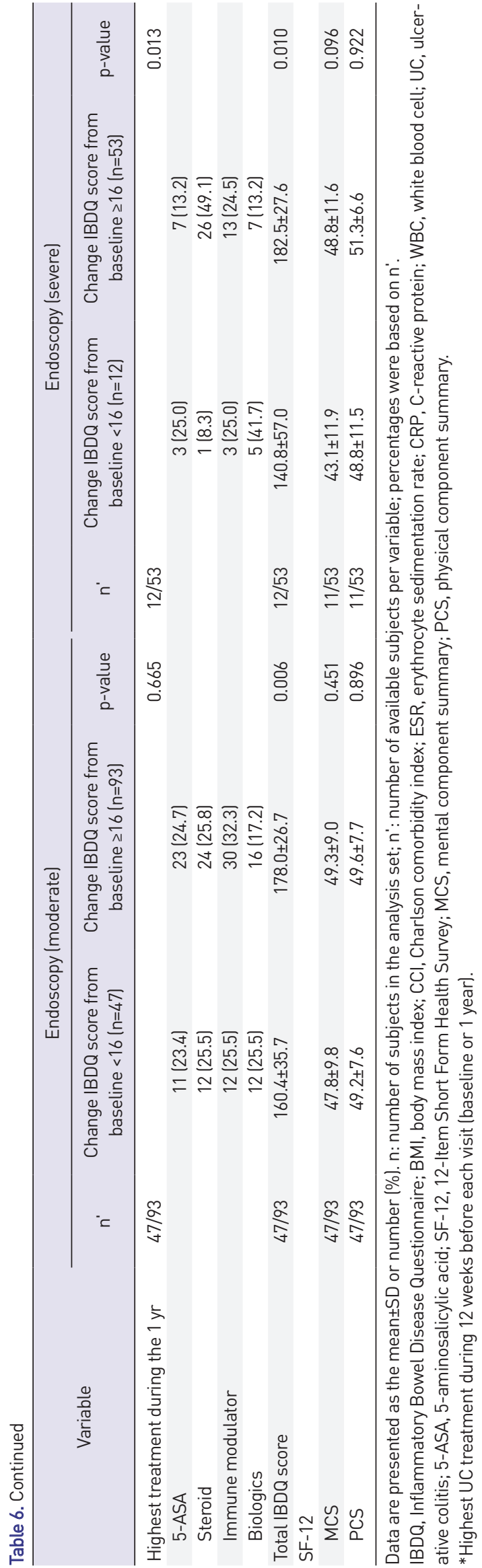

analyzing biologics administration and quality of life, it has been reported that biologics administration significantly improves the quality of life of patients. ${ }^{32-35}$

The partial Mayo score was identified as a possible predictor of HRQL in moderate-to-severe UC, and it showed a consistent tendency in both the IBDQ and SF-12. The relationship between disease activity and HRQL was dealt with in previous studies. A prospective study of mild-tomoderate UC patients reported a negative correlation between the IBDQ and the Mayo score. ${ }^{36}$ In addition, several studies have shown that the disabilities of UC patients, which closely affects the quality of life, worsened as the disease activity index increased. ${ }^{37-39}$ We also found correlations between inflammatory markers (CRP, ESR, and white blood cell count) and HRQL. As far as we know, there are few studies that have attempted to directly correlate laboratory findings and HRQL results. We found that a more accurate prediction could be made by integrating multiple laboratory findings rather than focusing on one specific item when trying to estimate the HRQL. Several studies have reported a correlation between fatigue level and ESR or CRP in UC patients, ${ }^{40,41}$ and some clinical trials suggested that the higher CRP group had a lower IBDQ score or more severe level of emotional anxiety and depression. ${ }^{42,43}$ These results indirectly show that inflammatory markers can reflect HRQL.

Women, older age, and a history of smoking or alcohol consumption were also correlated with poorer HRQL in our data. In other inflammatory bowel disease studies, females had a higher rate of poor HRQL like our results ${ }^{31,44}$ We thought this may be aroused from pregnancy and childbearing problems, as well as fewer opportunities in workplace and social activities. In patients of elderly IBD, the pattern in which the patient's age affects HRQL is unique. For example, there was a report that elderly patients have more severe deterioration in quality of life in terms of physical rather than mental aspect. ${ }^{45}$ Alcohol and smoking have been associated with poor quality of life in previous studies. ${ }^{38,46}$ Excessive alcohol consumption may have a negative effect in remission, so this point may be related to HRQL. ${ }^{47}$ A study reported active smoking may be associated with lower adherence to treatment, while other researchers have approached in terms of medical cost burden due to tobacco use. . $^{48,49}$

To the best of our knowledge, few studies have focused on changes in the HRQL of IBD patients in the early stages of disease. Since patients with mild UC at the time of diagnosis were excluded, we were able to minimize the effect of disease severity on HRQL. In addition, because the MOSAIK cohort was a well-organized, prospective cohort that was composed of single-country and Asian patients, 
confounders caused by regional and racial heterogeneity within the cohort were minimized. As for survey methodologies for HRQL, we chose a patient-reported on-site questionnaire. Patients were asked to fill out the questionnaire at their regular hospital visits. A medical staff member who was acquainted with the study contents in advance was present to assist the patient in filling out the questionnaire if necessary. Through this, the limitations of other survey methods such as low response rates and difficulty in controlling respondents could be overcome.

This study has some limitations. First, a fundamental limitation of the questionnaire-based survey exists. IBDQ and SF-12 were used at the regular follow-up appointments, and there was an inevitable time interval between real life and the point when the questionnaire was completed, which could provoke unpredictable interventions such as recall bias. Currently, app-based, daily selfreporting systems by patients themselves have been used for accurate evaluation of both disease and patient status in the clinical field. We hope future studies using newly invented methods will compensate for these methodological limitations. Second, since the population of the MOSAIK cohort mainly consists of Koreans, there is the possibility of racial, regional, or cultural bias; further, accessibility for diagnosis and treatment may be different from that of other countries. Lastly, we were unable to access "beforethe-diagnosis" factors such as time interval from the first manifestation to the diagnosis, which may have influence on HRQL in the early phase of the disease.

In conclusion, patients with moderate-to-severe UC showed significant improvement in HRQL over the course of 1 year after diagnosis when the disease activity is well controlled. In the future, the disease course of moderateto-severe UC patients and their HRQL fluctuations will require further long-term data accumulation and analysis.

\section{CONFLICTS OF INTEREST}

The authors of this publication have research support from Janssen Korea. No other potential conflict of interest relevant to this article was reported.

\section{ACKNOWLEDGEMENTS}

The authors acknowledge and would like to thank all members of the Moderate-to-Severe Ulcerative Colitis in Korea (MOSAIK) study group for participating in the study. We would like to thank Janssen Korea for sponsoring the study.

\section{AUTHOR CONTRIBUTIONS}

Study concept and design: K.M.L., D.B.K. Data acquisition: D.B.K., J.W.K., J.L., Y.T.J., T.O.K., J.S.K., J.J.P., S.N.H., D.I.P., H.S.K., Y.J.L. Data analysis and interpretation: I.H.J., K.M.L., D.B.K., Y.K. Drafting of the manuscript: I.H.J. Critical revision of the manuscript: I.H.J., K.M.L., D.B.K., J.W.K., J.L., Y.T.J., T.O.K., J.S.K., J.J.P., S.N.H., D.I.P., H.S.K., Y.J.L., Y.K. All authors approved the final submission.

\section{ORCID}

Ik Hyun Jo https://orcid.org/0000-0002-3433-8662

Kang-Moon Lee https://orcid.org/0000-0003-2850-4553

Dae Bum Kim https://orcid.org/0000-0003-0830-3375

Ji Won Kim https://orcid.org/0000-0002-1214-5544

Jun Lee https://orcid.org/0000-0002-8060-9646

Yoon Tae Jeen https://orcid.org/0000-0003-0220-3816

Tae-Oh Kim https://orcid.org/0000-0002-7359-1599

Joo Sung Kim https://orcid.org/0000-0001-6835-4735

Jae Jun Park https://orcid.org/0000-0001-9974-1658

Sung Noh Hong https://orcid.org/0000-0002-4140-3717

Dong Il Park https://orcid.org/0000-0003-2307-8575

Hyun-Soo Kim https://orcid.org/0000-0001-7190-0362

Yoo Jin Lee https://orcid.org/0000-0003-1799-0146

Youngdoe Kim https://orcid.org/0000-0002-0772-6360

\section{REFERENCES}

1. Baumgartner RN, Koehler KM, Gallagher D, et al. Epidemiology of sarcopenia among the elderly in New Mexico. Am J Epidemiol 1998;147:755-763.

2. Feuerstein JD, Moss AC, Farraye FA. Ulcerative colitis. Mayo Clin Proc 2019;94:1357-1373.

3. Trigo-Vicente C, Gimeno-Ballester V, García-López S, López-Del Val A. Systematic review and network meta-analysis of treatment for moderate-to-severe ulcerative colitis. Int J Clin Pharm 2018;40:1411-1419.

4. Singh S, Fumery M, Sandborn WJ, Murad MH. Systematic review with network meta-analysis: first- and second-line pharmacotherapy for moderate-severe ulcerative colitis. Aliment Pharmacol Ther 2018;47:162-175.

5. Kemp K, Dibley L, Chauhan U, et al. Second N-ECCO consensus statements on the European nursing roles in caring for patients with Crohn's disease or ulcerative colitis. J Crohns Colitis 2018;12:760-776.

6. Sarid O, Slonim-Nevo V, Schwartz D, et al. Differing relationship of psycho-social variables with active ulcerative colitis or Crohn's disease. Int J Behav Med 2018;25:341-350. 
7. Larsson K, Lööf L, Nordin K. Stress, coping and support needs of patients with ulcerative colitis or Crohn's disease: a qualitative descriptive study. J Clin Nurs 2017;26:648-657.

8. Moon JR, Lee CK, Hong SN, et al. Unmet psychosocial needs of patients with newly diagnosed ulcerative colitis: results from the nationwide prospective cohort study in Korea. Gut Liver 2020;14:459-467.

9. Daperno M, Armuzzi A, Danese S, et al. Unmet medical needs in the management of ulcerative colitis: results of an Italian Delphi consensus. Gastroenterol Res Pract 2019;2019:3108025.

10. Ho EY, Cominelli F, Katz J. Ulcerative colitis: what is the optimal treatment goal and how do we achieve it? Curr Treat Options Gastroenterol 2015;13:130-142.

11. Gajendran M, Loganathan P, Jimenez G, et al. A comprehensive review and update on ulcerative colitis. Dis Mon 2019;65:100851.

12. Rivett L, Rothberg A, Stewart A, Berkowitz R. The relationship between quality of life and compliance to a brace protocol in adolescents with idiopathic scoliosis: a comparative study. BMC Musculoskelet Disord 2009;10:5.

13. Yarur AJ, Strobel SG, Deshpande AR, Abreu MT. Predictors of aggressive inflammatory bowel disease. Gastroenterol Hepatol (N Y) 2011;7:652-659.

14. Lee CK, Lee KM, Park DI, et al. A new opportunity for innovative inflammatory bowel disease research: the Moderateto-Severe Ulcerative Colitis in Korea (MOSAIK) cohort study. Intest Res 2019;17:1-5.

15. D'Haens G, Sandborn WJ, Feagan BG, et al. A review of activity indices and efficacy end points for clinical trials of medical therapy in adults with ulcerative colitis. Gastroenterology 2007;132:763-786.

16. Kim WH, Cho YS, Yoo HM, Park IS, Park EC, Lim JG. Quality of life in Korean patients with inflammatory bowel diseases: ulcerative colitis, Crohn's disease and intestinal Behçet's disease. Int J Colorectal Dis 1999;14:52-57.

17. Feagan BG, Patel H, Colombel JF, et al. Effects of vedolizum$\mathrm{ab}$ on health-related quality of life in patients with ulcerative colitis: results from the randomised GEMINI 1 trial. Aliment Pharmacol Ther 2017;45:264-275.

18. Gregor JC, McDonald JW, Klar N, et al. An evaluation of utility measurement in Crohn's disease. Inflamm Bowel Dis 1997;3:265-276.

19. Huoponen S, Eberl A, Räsänen P, et al. Health-related quality of life and costs of switching originator infliximab to biosimilar one in treatment of inflammatory bowel disease. Medicine (Baltimore) 2020;99:e18723.

20. Ware J Jr, Kosinski M, Keller SD. A 12-Item Short-Form Health Survey: construction of scales and preliminary tests of reliability and validity. Med Care 1996;34:220-233.

21. Busija L, Pausenberger E, Haines TP, Haymes S, Buchbinder
$\mathrm{R}$, Osborne RH. Adult measures of general health and health-related quality of life: medical outcomes Study Short Form 36-Item (SF-36) and Short Form 12-Item (SF-12) Health Surveys, Nottingham Health Profile (NHP), Sickness Impact Profile (SIP), Medical Outcomes Study Short Form 6D (SF-6D), Health Utilities Index Mark 3 (HUI3), Quality of Well-Being Scale (QWB), and Assessment of Quality of Life (AQoL). Arthritis Care Res (Hoboken) 2011;63 Suppl 11:S383-S412.

22. Gandek B, Ware JE, Aaronson NK, et al. Cross-validation of item selection and scoring for the SF-12 Health Survey in nine countries: results from the IQOLA Project. International Quality of Life Assessment. J Clin Epidemiol 1998;51:1171-1178.

23. Stepaniuk P, Bernstein CN, Nugent Z, Singh H. Characterization of inflammatory bowel disease in elderly hospitalized patients in a large central Canadian Health region. Can J Gastroenterol Hepatol 2015;29:274-278.

24. Burisch J, Katsanos KH, Christodoulou DK, et al. Natural disease course of ulcerative colitis during the first five years of follow-up in a European population-based inception cohort: an Epi-IBD study. J Crohns Colitis 2019;13:198-208.

25. Müller O, Baumann C, Di Patrizio P, et al. Patient's early satisfaction with care: a predictor of health-related quality of life change among outpatients with substance dependence. Health Qual Life Outcomes 2020;18:6.

26. Burisch J, Weimers P, Pedersen N, et al. Health-related quality of life improves during one year of medical and surgical treatment in a European population-based inception cohort of patients with inflammatory bowel disease: an ECCOEpiCom study. J Crohns Colitis 2014;8:1030-1042.

27. Cao Q, Huang YH, Jiang M, Dai C. The prevalence and risk factors of psychological disorders, malnutrition and quality of life in IBD patients. Scand J Gastroenterol 2019;54:14581466.

28. Fourie S, Jackson D, Aveyard H. Living with inflammatory bowel disease: a review of qualitative research studies. Int J Nurs Stud 2018;87:149-156.

29. Knowles SR, Graff LA, Wilding H, Hewitt C, Keefer L, Mikocka-Walus A. Quality of life in inflammatory bowel disease: a systematic review and meta-analyses. Part I. Inflamm Bowel Dis 2018;24:742-751.

30. Christiansen LK, Lo B, Bendtsen F, Vind I, Vester-Andersen $\mathrm{MK}$, Burisch J. Health-related quality of life in inflammatory bowel disease in a Danish population-based inception cohort. United European Gastroenterol J 2019;7:942-954.

31. Huppertz-Hauss G, Lie Høivik M, Jelsness-Jørgensen LP, et al. Health-related quality of life in patients with inflammatory bowel disease 20 years after diagnosis: results from the IBSEN study. Inflamm Bowel Dis 2016;22:1679-1687.

32. Sandborn WJ, Feagan BG, Marano C, et al. Subcutaneous 
golimumab induces clinical response and remission in patients with moderate-to-severe ulcerative colitis. Gastroenterology 2014;146:85-95.

33. Loftus EV Jr, Colombel JF, Feagan BG, et al. Long-term efficacy of vedolizumab for ulcerative colitis. J Crohns Colitis 2017;11:400-411.

34. Sparrow MP. Adalimumab in ulcerative colitis: efficacy, safety and optimization in the era of treat-to target. Expert Opin Biol Ther 2017;17:613-621.

35. Fernández-Clotet A, Castro-Poceiro J, Panés J. Tofacitinib for the treatment of ulcerative colitis. Expert Rev Clin Immunol 2018;14:881-892.

36. van der Have M, Brakenhoff LK, van Erp SJ, et al. Back/joint pain, illness perceptions and coping are important predictors of quality of life and work productivity in patients with inflammatory bowel disease: a 12-month longitudinal study. J Crohns Colitis 2015;9:276-283.

37. Leong RW, Huang T, Ko Y, et al. Prospective validation study of the International Classification of Functioning, Disability and Health score in Crohn's disease and ulcerative colitis. J Crohns Colitis 2014;8:1237-1245.

38. Marinelli C, Zingone F, Inferrera M, et al. Factors associated with disability in patients with ulcerative colitis: a crosssectional study. J Dig Dis 2020;21:81-87.

39. Magalhães J, Castro FD, Carvalho PB, et al. Disability in inflammatory bowel disease: translation to Portuguese and validation of the "Inflammatory Bowel Disease - Disability Score". GE Port J Gastroenterol 2015;22:4-14.

40. Graff LA, Vincent N, Walker JR, et al. A population-based study of fatigue and sleep difficulties in inflammatory bowel disease. Inflamm Bowel Dis 2011;17:1882-1889.

41. Yoo S, Jung YS, Park JH, et al. Fatigue severity and factors associated with high fatigue levels in Korean patients with inflammatory bowel disease. Gut Liver 2014;8:148-153.

42. Sadeghi N, Mansoori A, Shayesteh A, Hashemi SJ. The effect of curcumin supplementation on clinical outcomes and inflammatory markers in patients with ulcerative colitis. Phytother Res 2020;34:1123-1133.

43. Abautret-Daly Á, Dempsey E, Riestra S, et al. Association between psychological measures with inflammatory anddisease-related markers of inflammatory bowel disease. Int J Psychiatry Clin Pract 2017;21:221-230.

44. Slonim-Nevo V, Sarid O, Friger M, et al. Effect of psychosocial stressors on patients with Crohn's disease: threatening life experiences and family relations. Eur J Gastroenterol Hepatol 2016;28:1073-1081.

45. Velonias G, Conway G, Andrews E, et al. Older age- and health-related quality of life in inflammatory bowel diseases. Inflamm Bowel Dis 2017;23:283-288.

46. Kamp KJ, Stommel M. Health-related quality of life among patients with inflammatory bowel disease: a case control study. Gastroenterol Nurs 2021;44:21-30.

47. Owczarek D, Rodacki T, Domagała-Rodacka R, Cibor D, Mach T. Diet and nutritional factors in inflammatory bowel diseases. World J Gastroenterol 2016;22:895-905.

48. Severs M, Mangen MJ, van der Valk ME, et al. Smoking is associated with higher disease-related costs and lower health-related quality of life in inflammatory bowel disease. J Crohns Colitis 2017;11:342-352.

49. Bruna-Barranco I, LuéA, Gargallo-Puyuelo CJ, et al. Young age and tobacco use are predictors of lower medication adherence in inflammatory bowel disease. Eur J Gastroenterol Hepatol 2019;31:948-953. 